\title{
Research on Control Algorithm of Electric Linear Loading System
}

\author{
Jianjie Lei ${ }^{1}$, Yuanxun Fan ${ }^{1}$, Weidong Pan ${ }^{1}$, Dejia $\mathrm{Tang}^{2}$, Jian $\mathrm{Tao}^{2}$ and Zhiwei $\mathrm{Xu}^{2}$ \\ ${ }^{1}$ School of Mechanical and Electrical Engineering, Nanjing University of Science and Technology, NanJing, China \\ ${ }^{2}$ Shanghai Aerospace Control Technology Institute, Shanghai, China
}

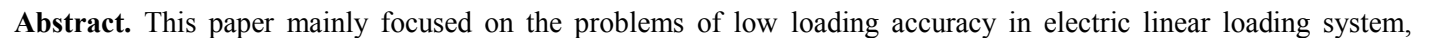

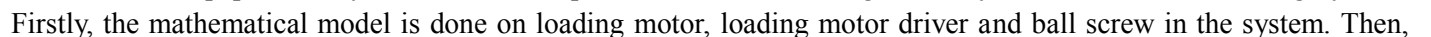

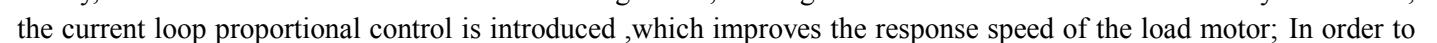
प

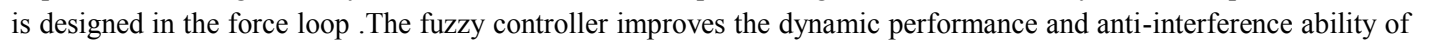

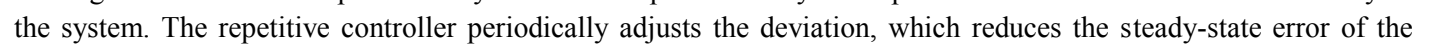

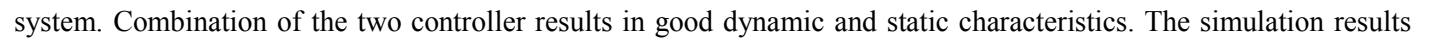

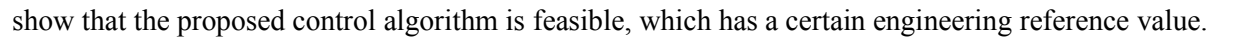

\section{Introduction}

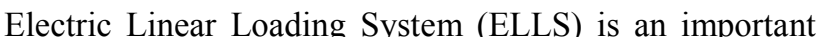

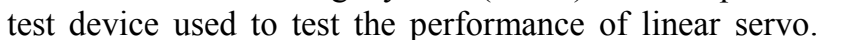

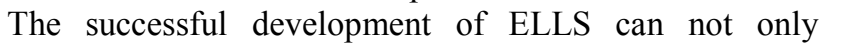

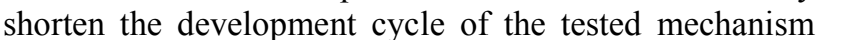

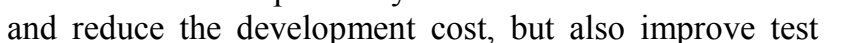

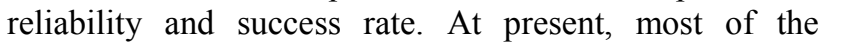

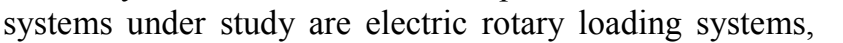

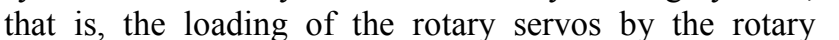

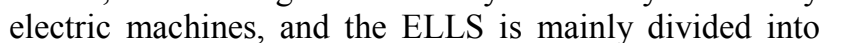

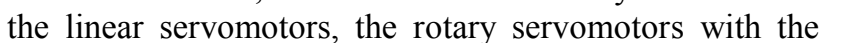

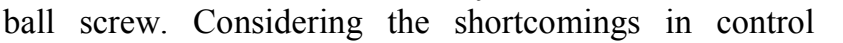

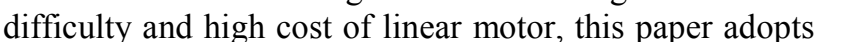

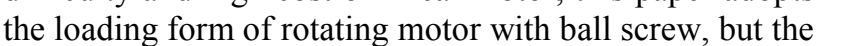

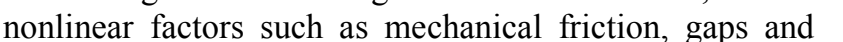

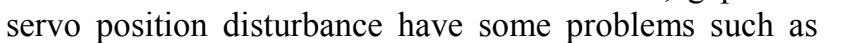

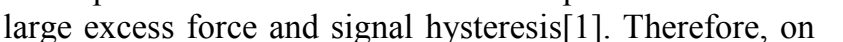

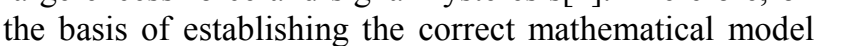

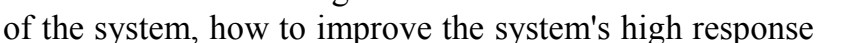

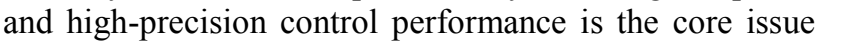

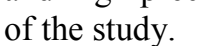

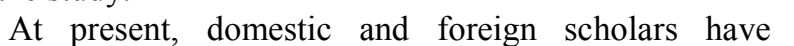

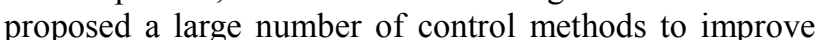

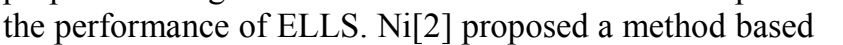

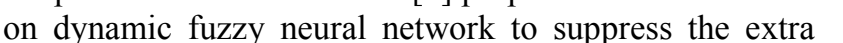

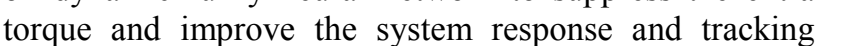

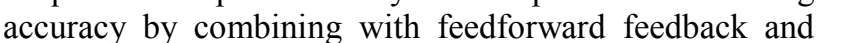

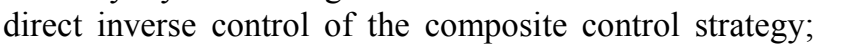

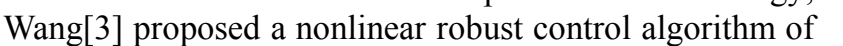

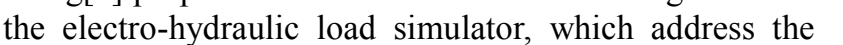
$\square$ tuator's disturbance and flow nonlinearity. Wang[4]

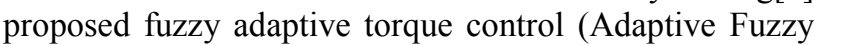

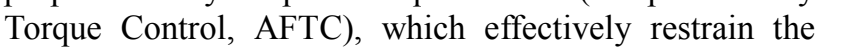

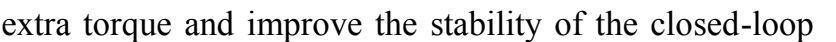

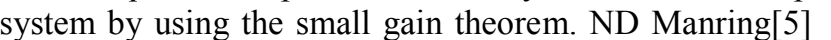

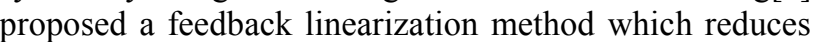

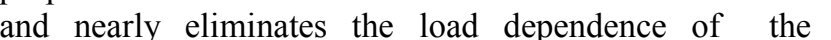

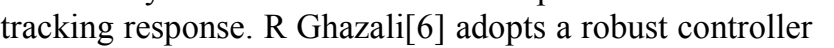

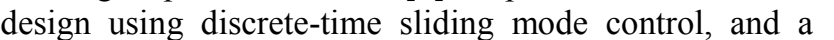

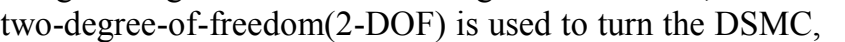

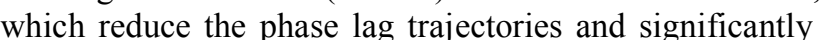

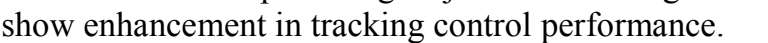

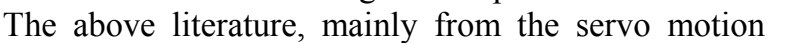

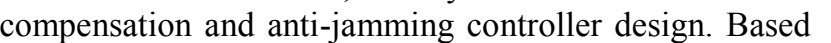

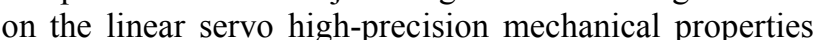

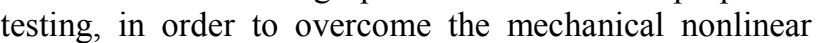

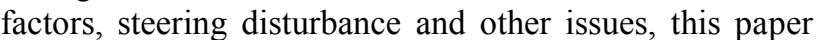

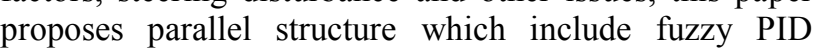

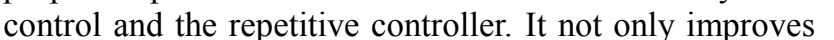

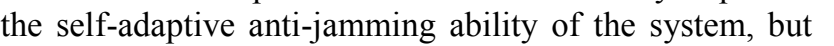

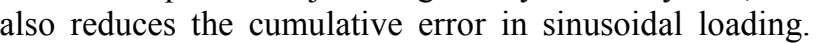

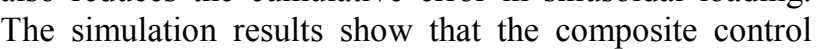

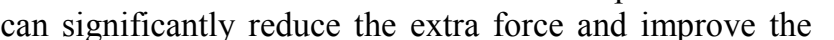

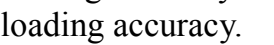

\section{System structure}

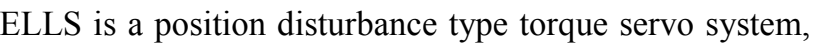

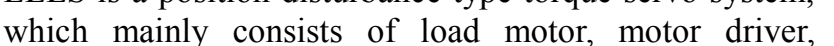

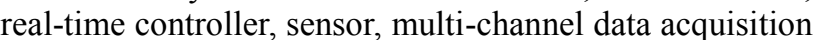

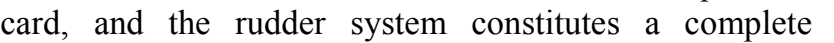

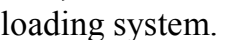

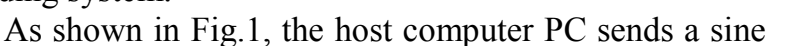

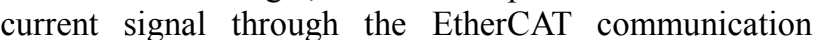

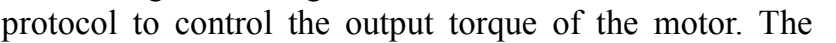
ए। 


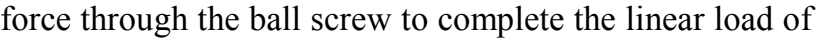

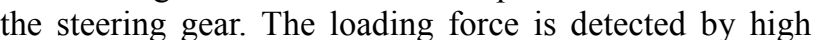

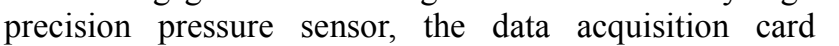

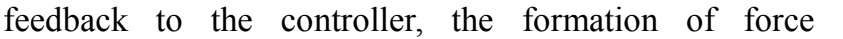

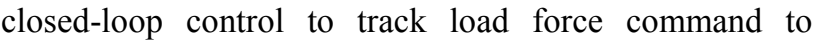

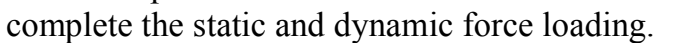

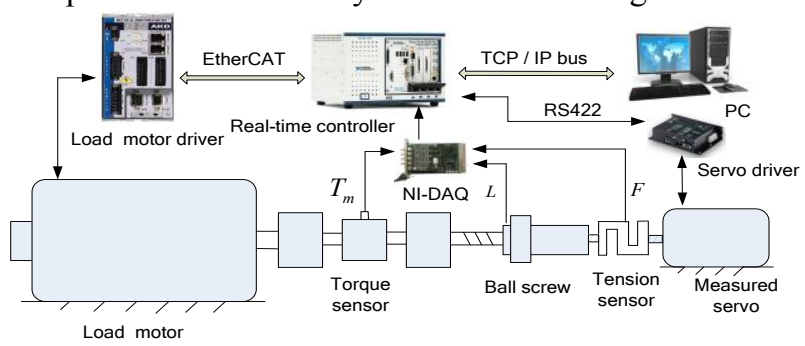

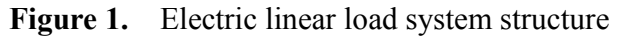

\section{System mathematical model}

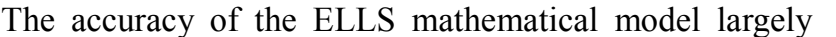

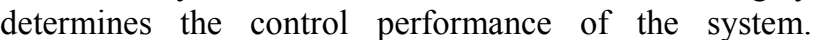

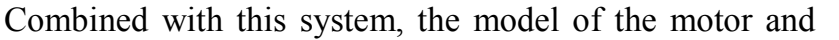

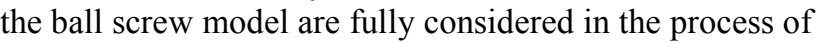

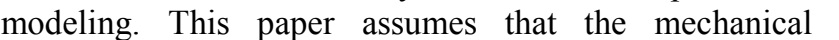

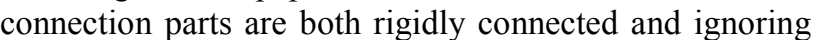

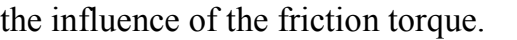

\subsection{Load motor model}

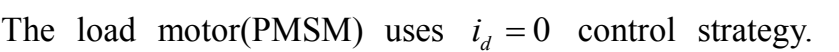

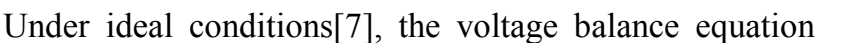

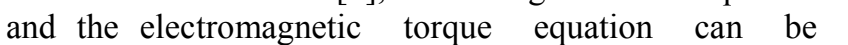

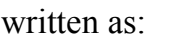

$$
\left.\begin{array}{c}
\square\|\|\|\| \square \\
\square_{q}=R_{a} i_{q}+L_{a} d i_{q} / d t+P \varphi_{f} W_{m} \\
J_{a} d W_{m} / d t=T_{e}-T_{L}-B_{a} W_{m}
\end{array}\right\} \square\|\|\|\| \| \square
$$
एवाण

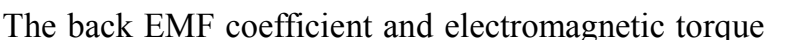

$$
\left.\begin{array}{l}
K_{e}=P \varphi_{f} \\
T_{e}=P \varphi_{f} i_{q}
\end{array}\right\} \square\|\|\|\|\|\|\|\|\|\| \|
$$

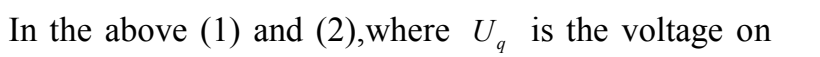

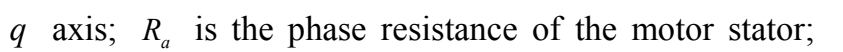

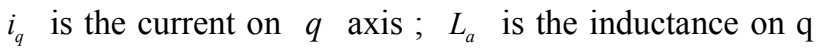

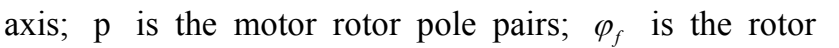

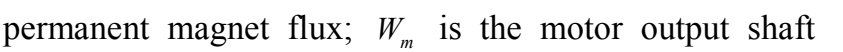

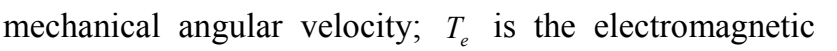

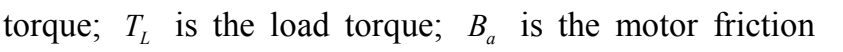

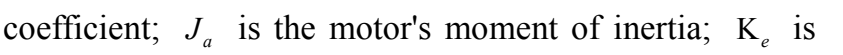

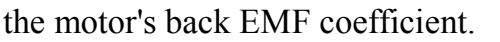

\subsection{Intermediate transformation model}

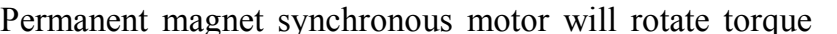

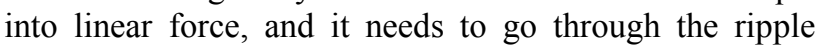

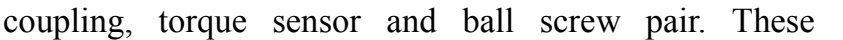

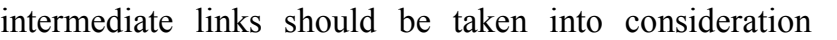

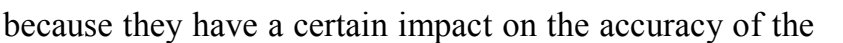

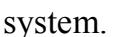

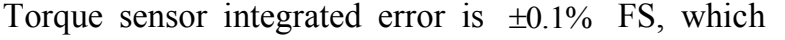
meets the Hulk's law: $\square$

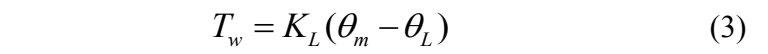

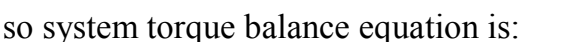

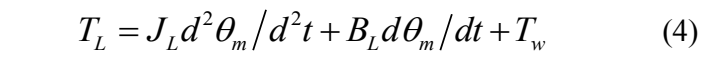

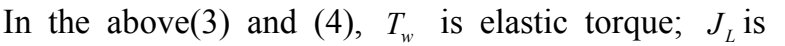

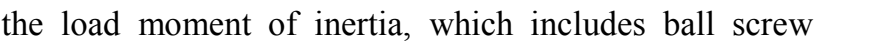

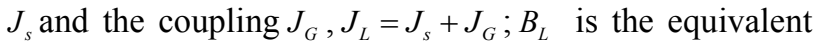

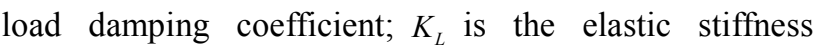

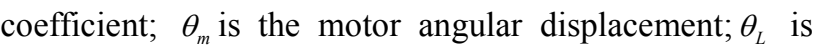

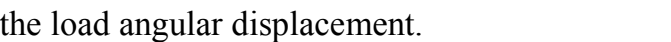

\subsection{Torque and force relationship of ball screw}

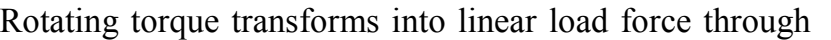

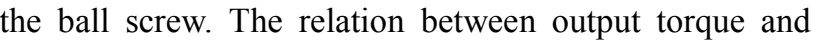

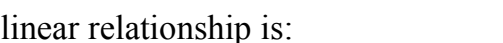

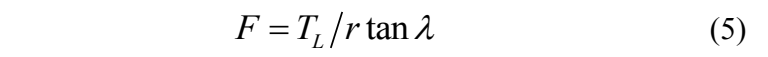

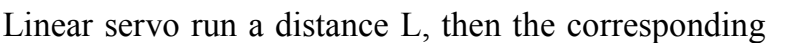

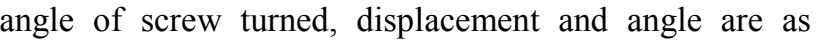

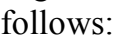

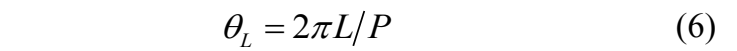

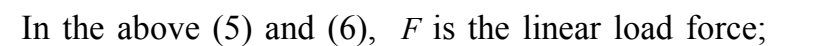

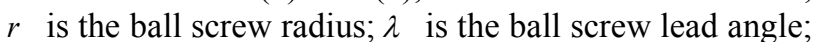

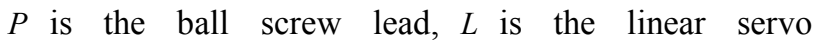

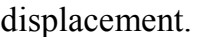

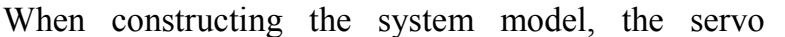

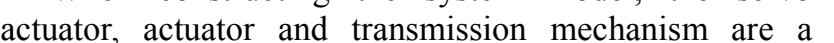

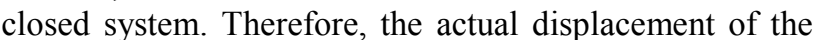

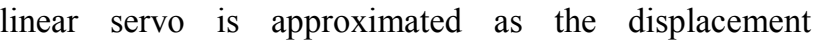

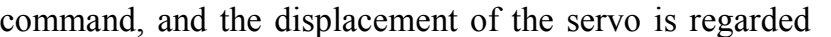

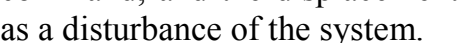

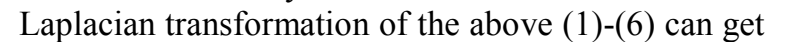

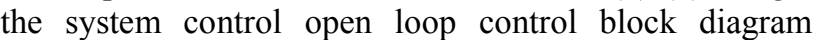

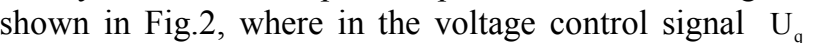

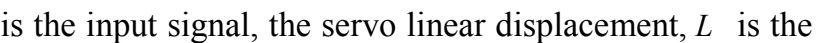

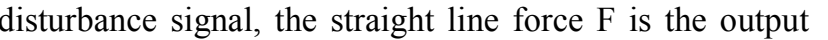
पाणाणामा

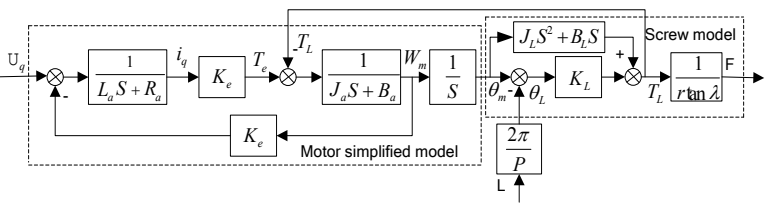

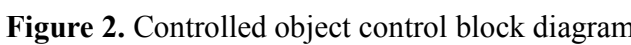

\section{Composite controller designer}

\subsection{Current loop design}

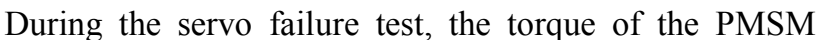

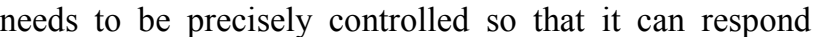

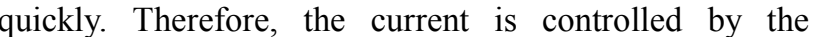




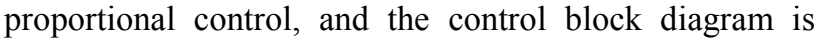

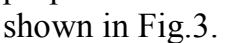

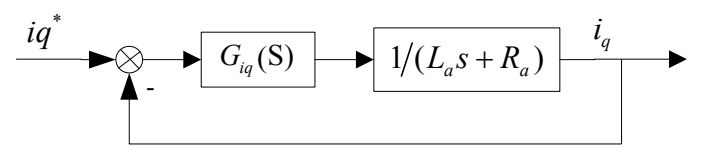

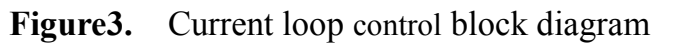

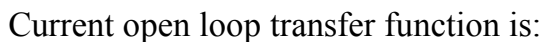

$$
G_{\square} \square \square=\square_{i q} \square \square \square_{a} \square+\square_{a} \square
$$

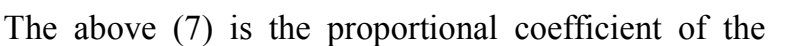

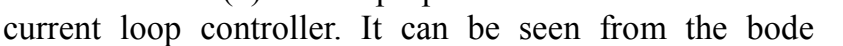

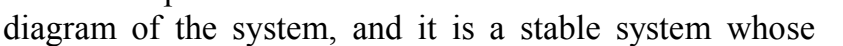

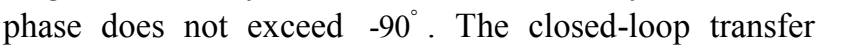

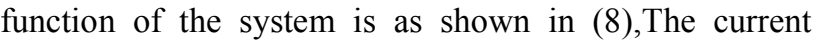

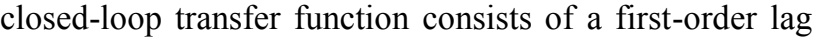

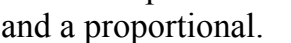

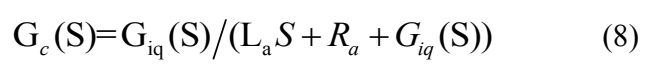

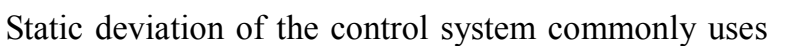

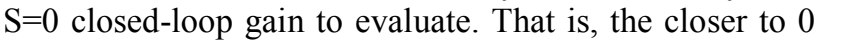

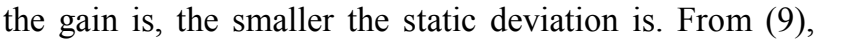

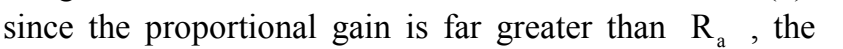

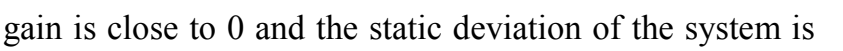
$\square \square$

$$
\square_{\varpi}(0) \square i_{q} \boxplus / i_{q}^{\square} \square \amalg=K_{i q} / \square_{a}+\square_{i q} \square 1111 \square \square
$$

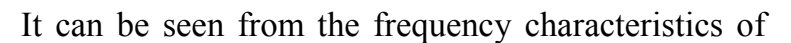

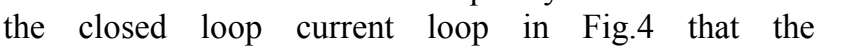

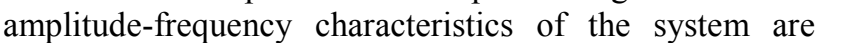

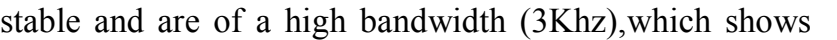

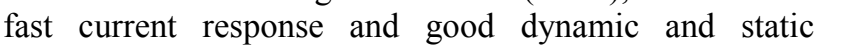

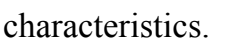

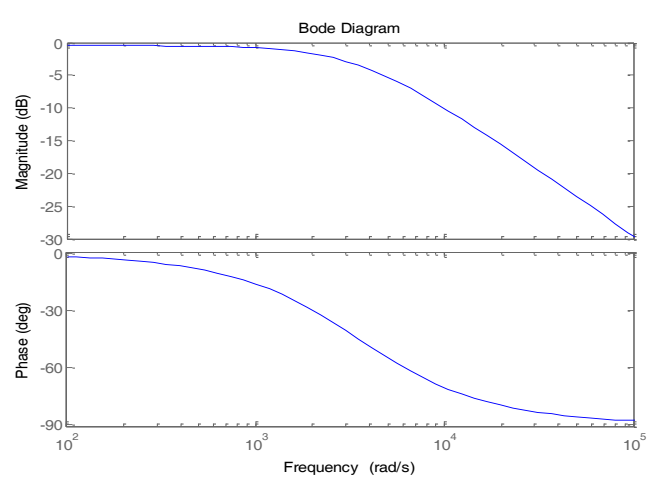

Figure.4

\subsection{Force controller design}

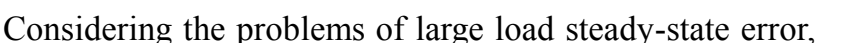

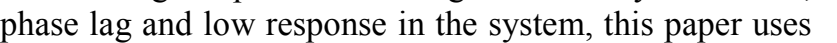

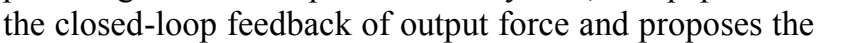

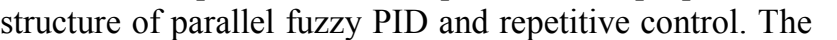

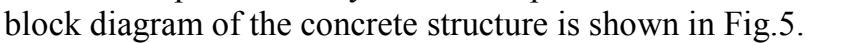

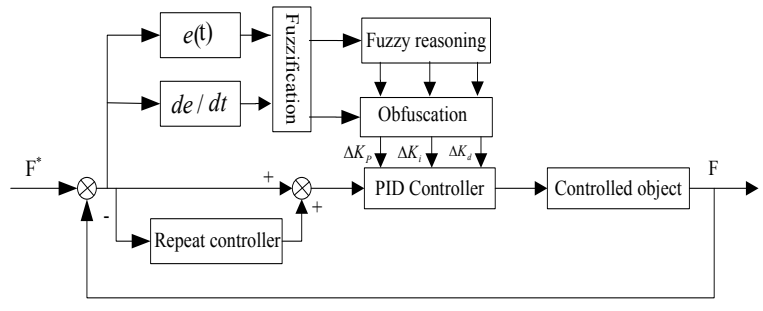

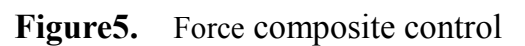

\subsubsection{Fuzzy PID Controller Design}

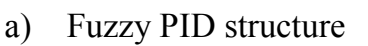

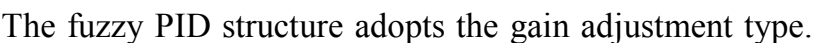

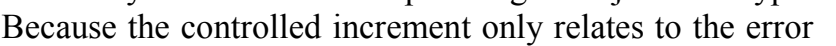

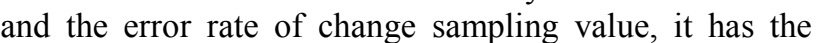

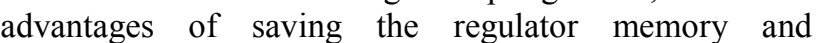

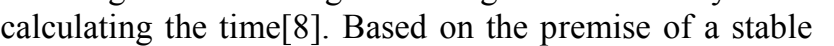

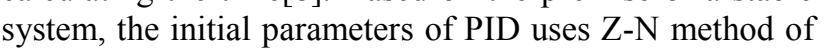

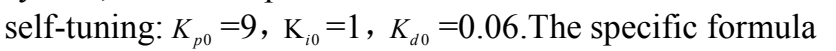
पा山ाए

$$
\left.\begin{array}{l}
K_{p} \amalg=\square_{p \square}+\Delta K_{p} \square \\
K_{i} \amalg=\square_{i \square}+\Delta K_{i} \square \square \\
K_{d} \square \amalg=\square_{d \square}+\Delta K_{d} \square
\end{array}\right\}
$$

एाए

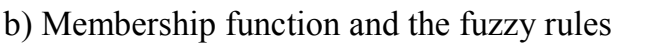

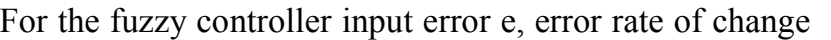

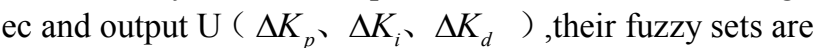

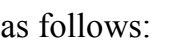

$$
\square \square \square=\{\mathrm{NB}, \mathrm{NM}, \mathrm{NS}, \mathrm{ZO}, \mathrm{PS}, \mathrm{PM}, \mathrm{PB}\}
$$

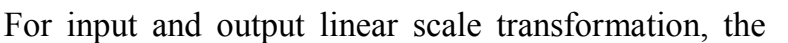

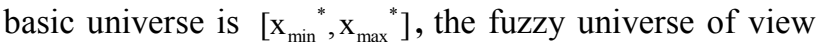

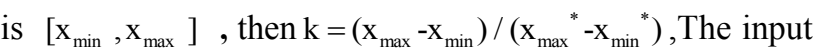

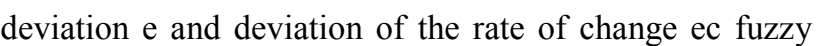

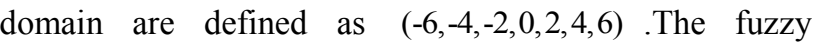

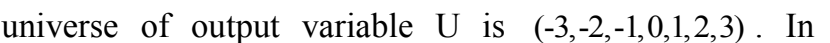

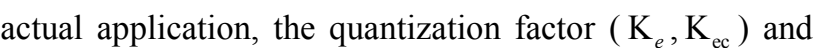

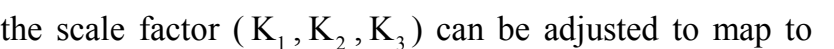

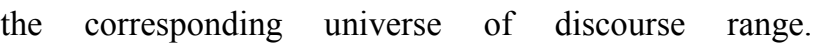

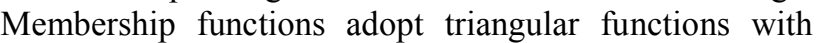

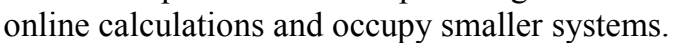

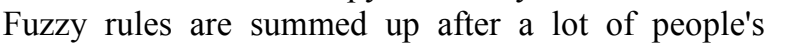

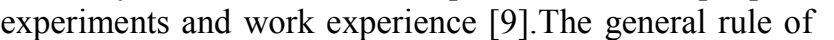

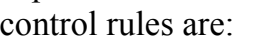

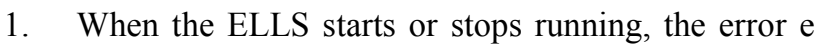

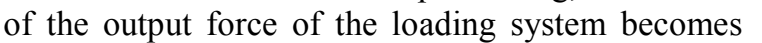

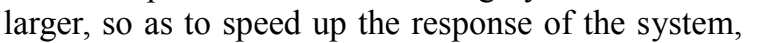

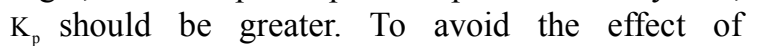

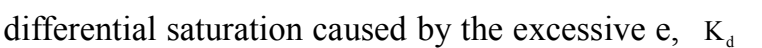

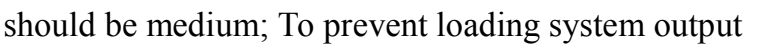

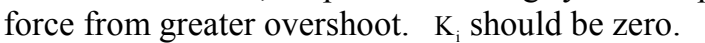

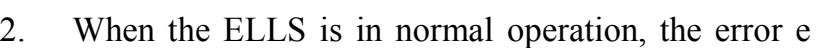




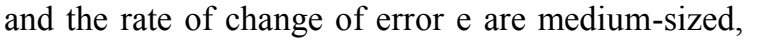

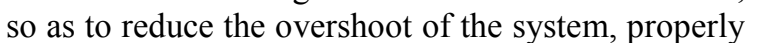

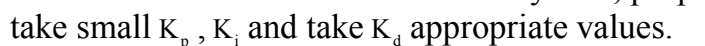

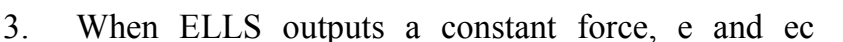

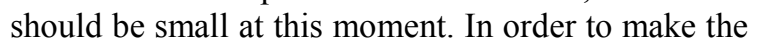

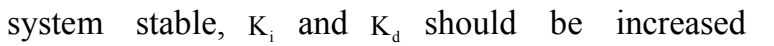

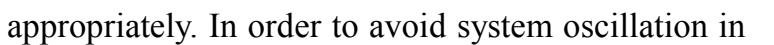

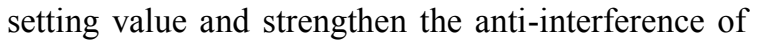

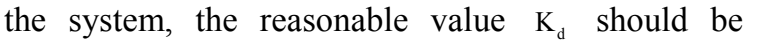
पा1णा
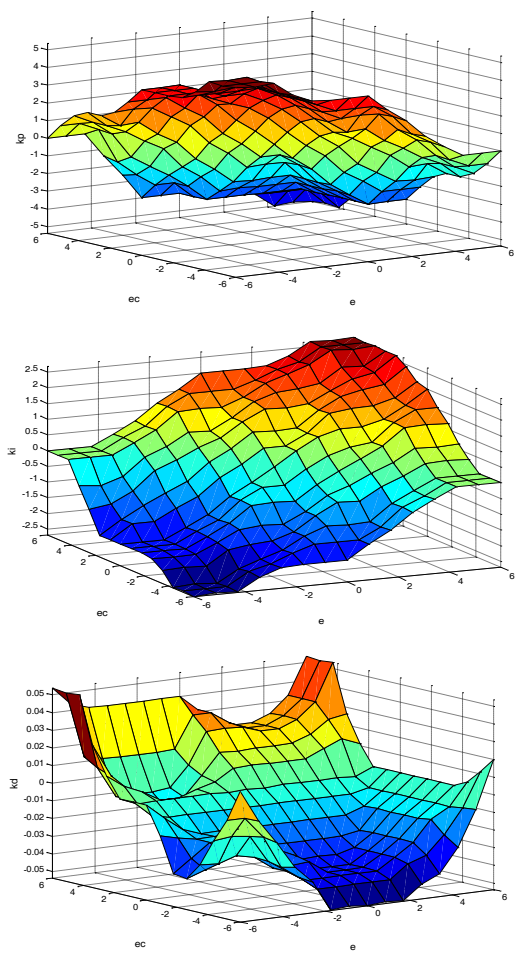

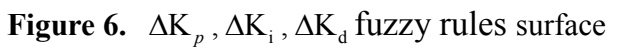

\section{पणमणणाणाप}

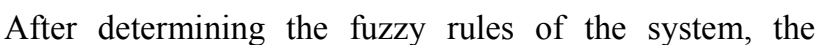

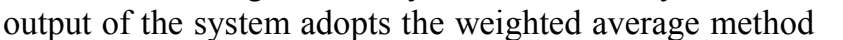

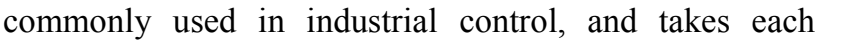

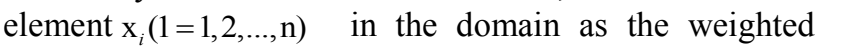
एण

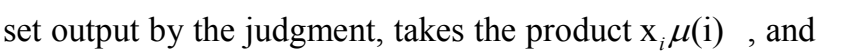

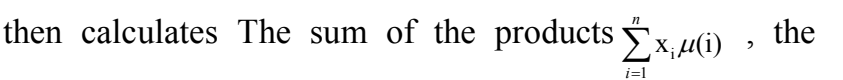

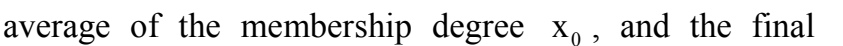
एயா

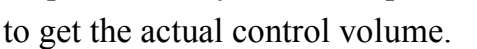

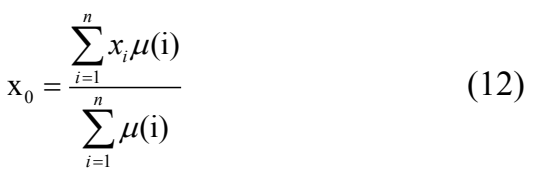

\subsubsection{Repetitive controller design}

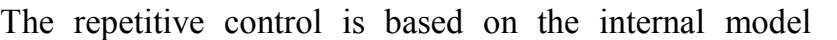

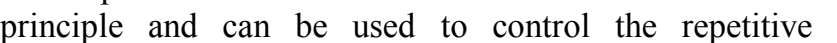

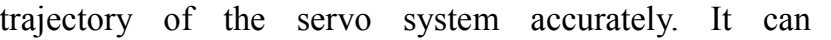

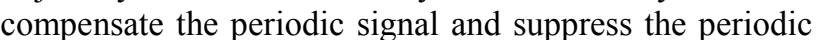

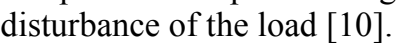

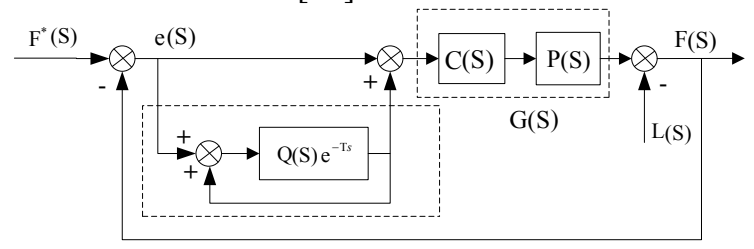

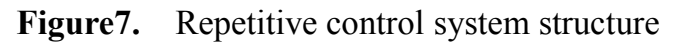

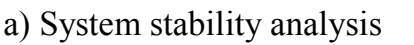

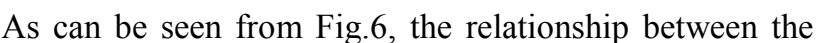
एण

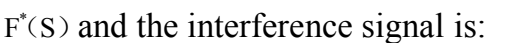

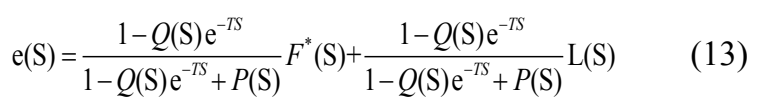

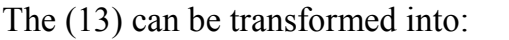

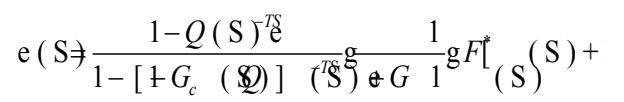

$\square \square \square$

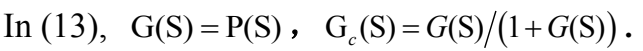

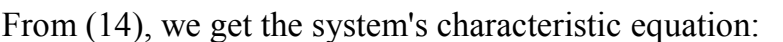

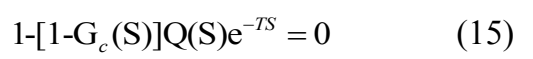

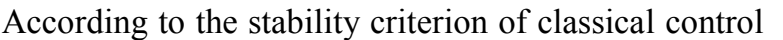

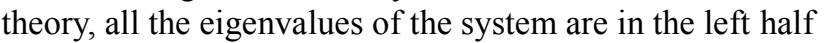

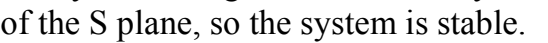

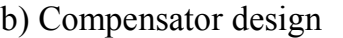

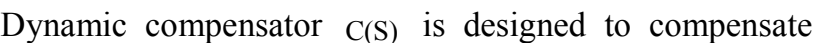
ए एव

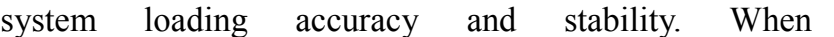

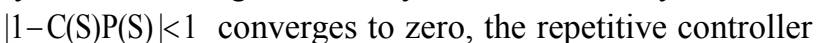

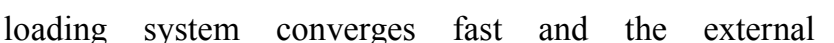

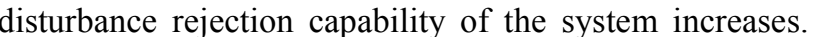

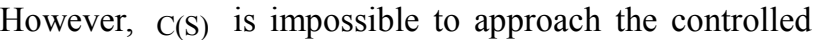

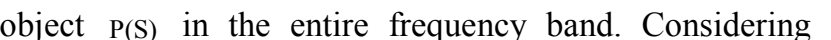
एம1

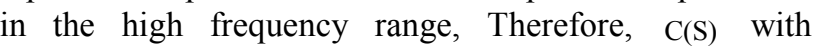

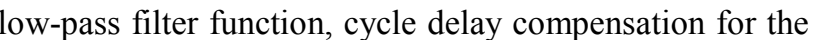

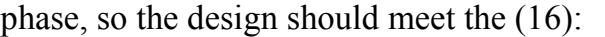

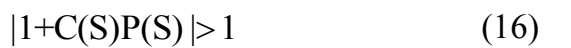

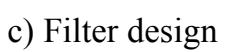

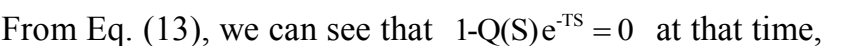
ए एव

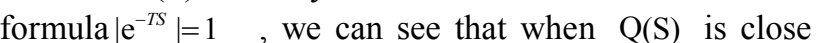

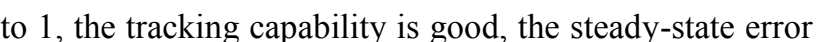

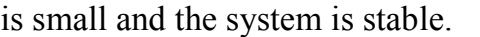

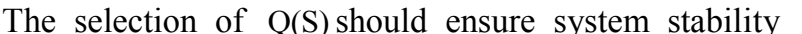

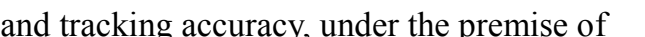




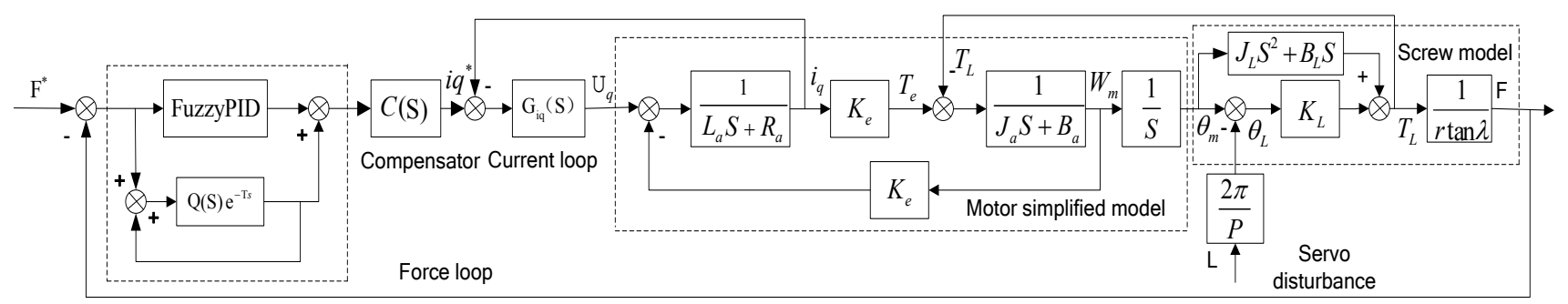

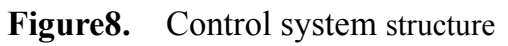

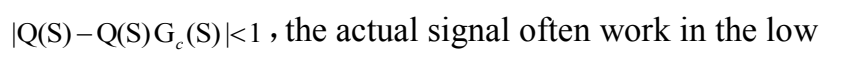

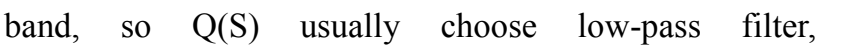

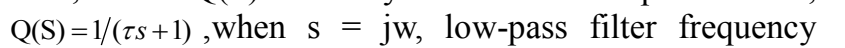

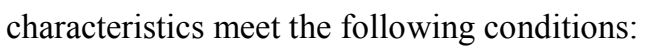

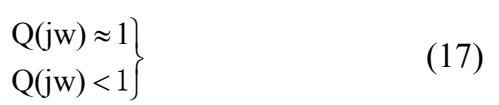

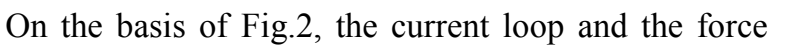

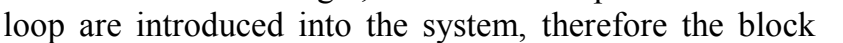

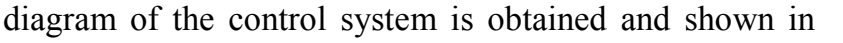
$\square[1 \square$

\section{System simulation}

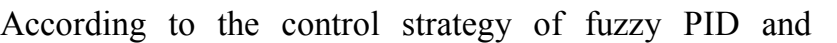

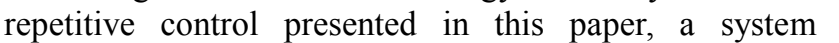

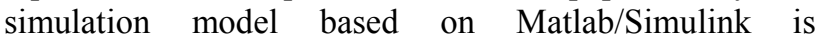

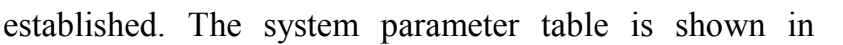

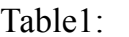

Table1. System parameters

\begin{tabular}{|c|c|c|c|}
\hline 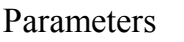 & 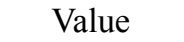 & $\square \square\|\mathbb{\|}\| \mathbb{\|} \|$ & $\square \square \square \square$ \\
\hline$K_{e} \square$ & $\square V \square K r p m \square$ & $r \square$ & $\square \Pi \mathrm{mm} \square$ \\
\hline$J_{L} \square$ & $\square \times \square{ }^{\square} \mathrm{kg}^{\square} \square$ & $\lambda \square$ & $\square \pi \mathbb{P}^{\circ} \square$ \\
\hline$B_{L} \square$ & 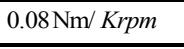 & $L_{a} \square$ & $\square \square m H \square$ \\
\hline$K_{L} \square$ & $\square \square N m \sqsubset$ rad $\square$ & $R_{m} \square$ & $\square \Omega \square$ \\
\hline$J_{m} \square$ & $\square \square \square \square \mathrm{Kgm} \square$ & $\tau \square$ & एणाए \\
\hline$B_{m} \square$ & 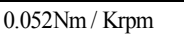 & $P \square$ & $\square \mathrm{mm} \square$ \\
\hline$\square_{\square} \square$ & $\square \square \square$ & $\square_{\square} \square$ & $\square \square$ \\
\hline$\square_{\square} \square$ & $\square$ & $\square_{\square} \square$ & $\square$ \\
\hline$\square_{\square} \square$ & एाए & $\square_{i q} \square$ & $\square$ \\
\hline
\end{tabular}

\subsection{Linear load force tracking performance}

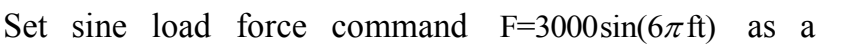

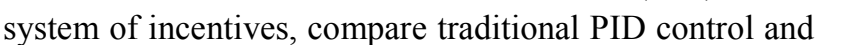

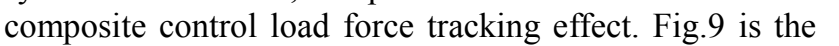

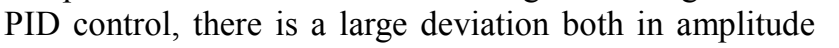

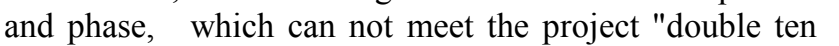

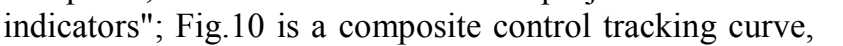

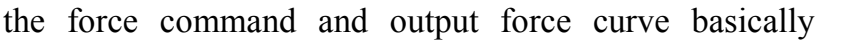

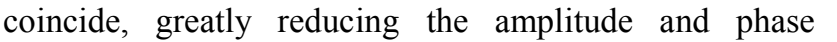

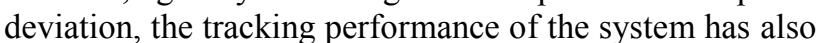

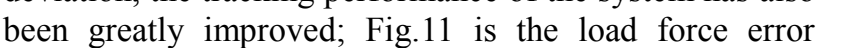

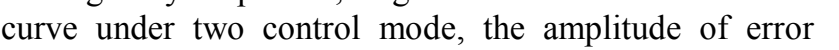

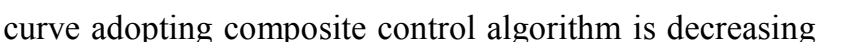

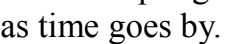

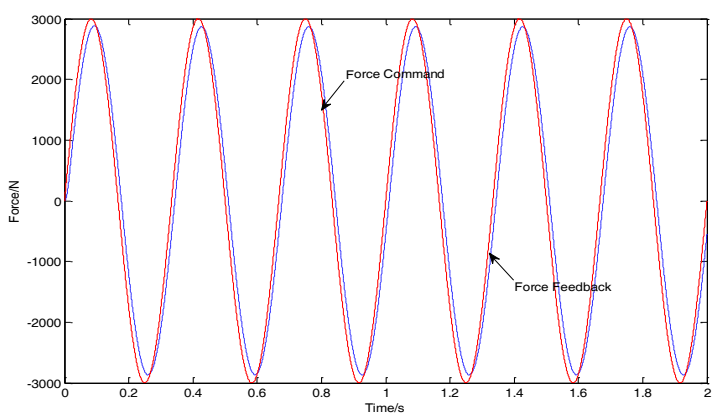

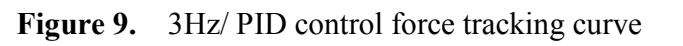

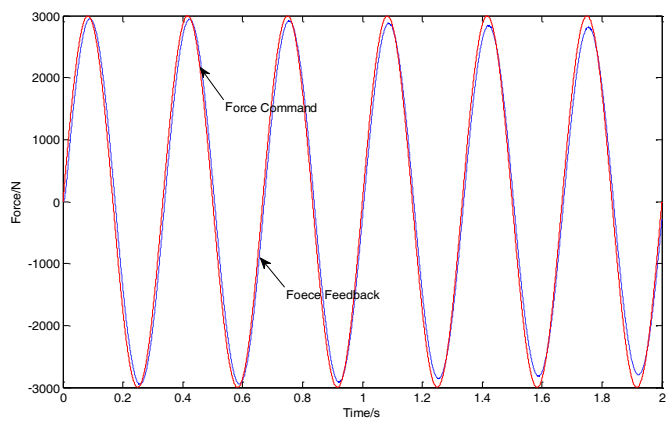

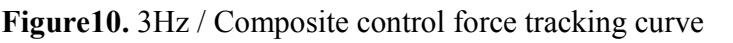

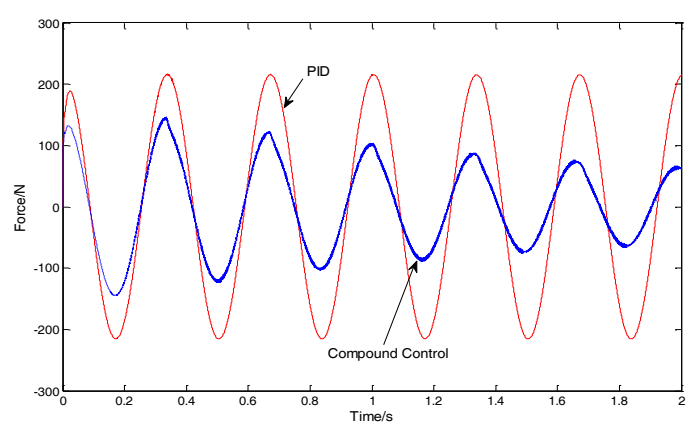

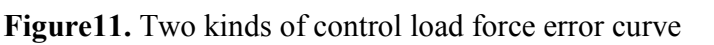

\subsection{Excess force suppression ability}

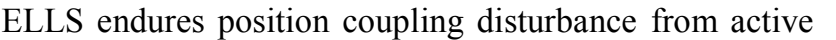

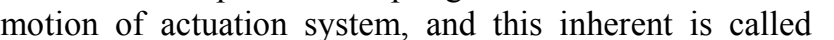

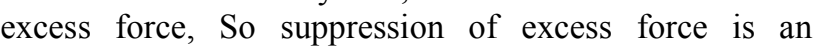

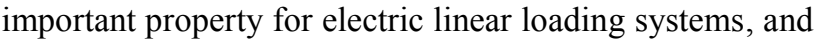

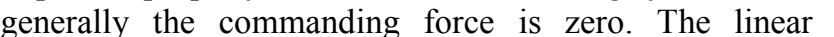




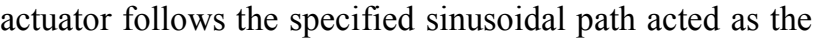

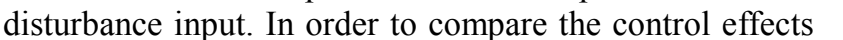

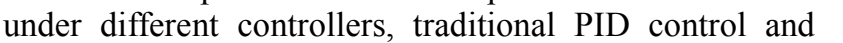

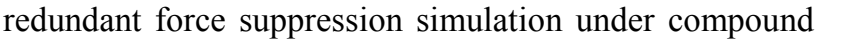

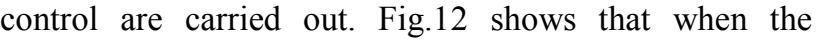

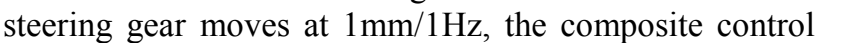

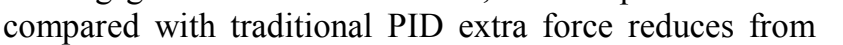

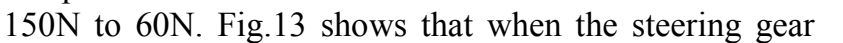

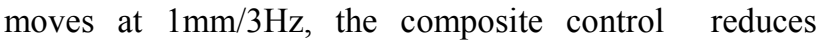

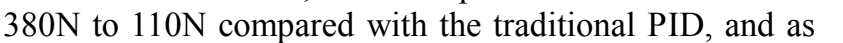

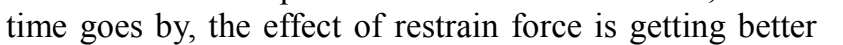

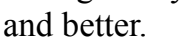

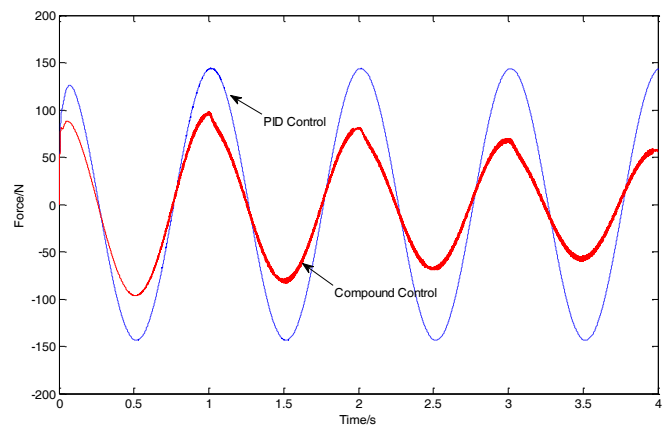

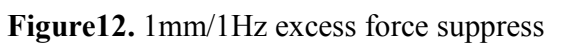

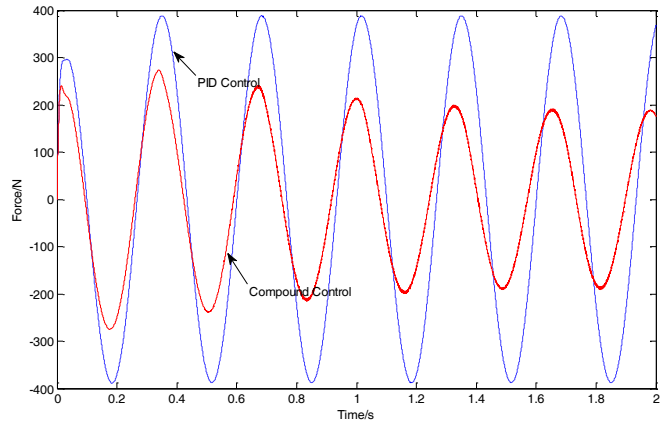

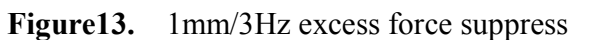

\section{Conclusion}

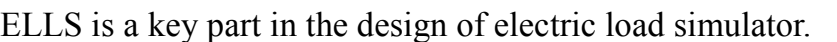

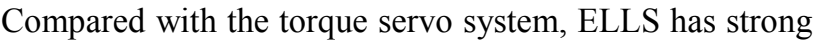

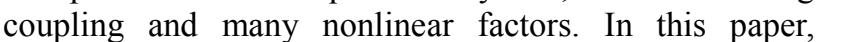

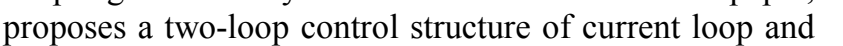

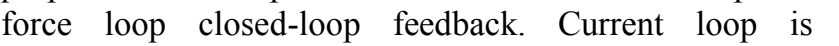

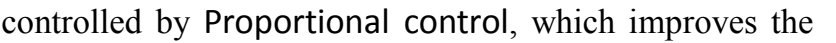

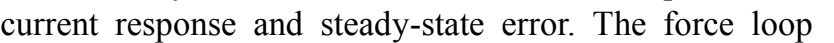

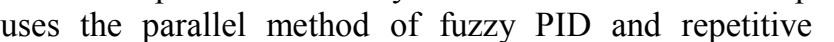

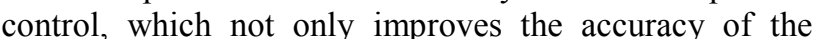

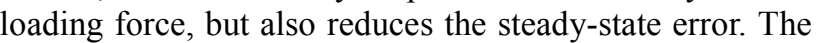

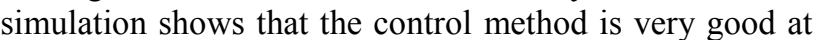

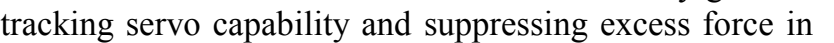

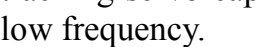

\section{References}

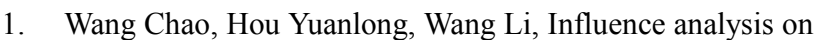

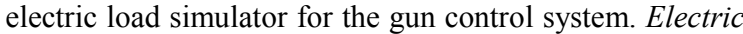
Machines and Control $\square \longrightarrow \mid \mathbf{2 0}(\mathbf{1 2}) \square \square \| \square \square$

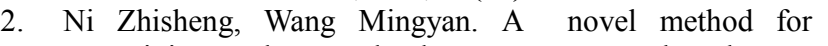

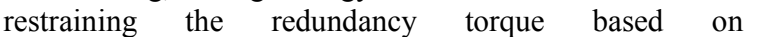
$\square \square \square \square \square$ Journal of harbin institute of technology $\square|| \mathbf{4 4}(\mathbf{1 0}) \square|\|\mid\|$

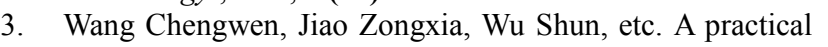

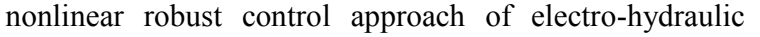

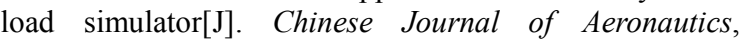

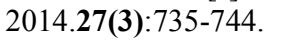

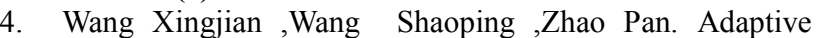

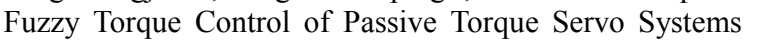

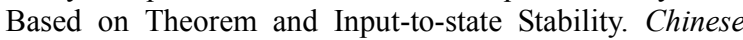
Journal of Aeronautics $\square \longrightarrow|\mathbf{2 5}(\mathbf{1 2}) \square||\square||| \mid$

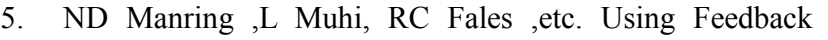

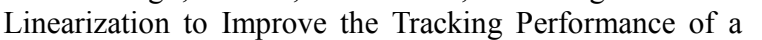

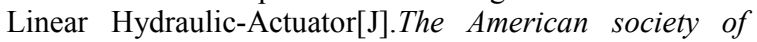

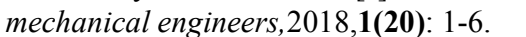

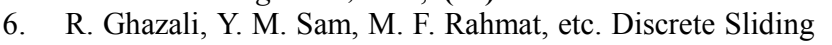

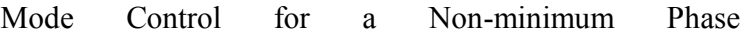

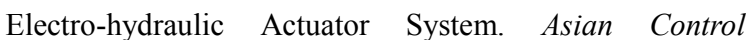
Conference (ASCC) प1माणाய

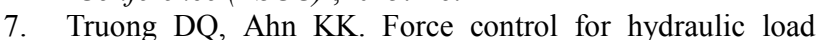

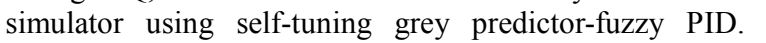
Mechatronics $\square|| \mathbf{1 9}(2) \square|||| \mid \square$

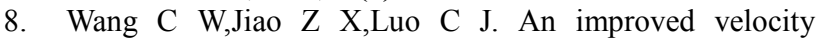

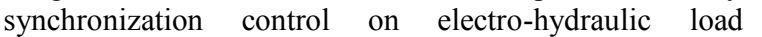

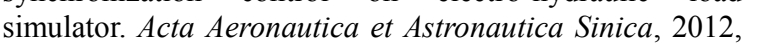
33(9) $\square|||| \||||| \mid$

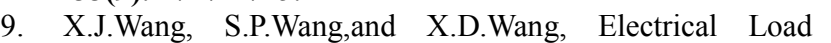

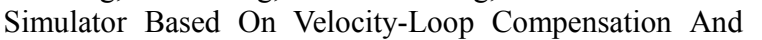

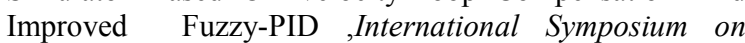
Industrial Electronics $\square \square 1 \square$

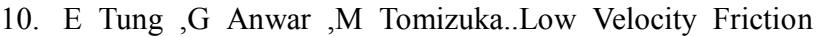

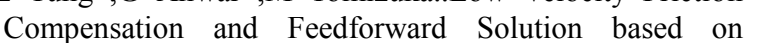

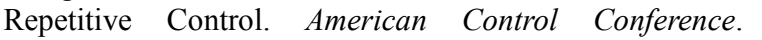
$\square\|\| \mathbf{1 1 5}(2) \square\|\| \mid\|\| \| \square \square$ 